\title{
GRADING OF TOMATOES USING DIGITAL IMAGE PROCESSING ON THE BASIS OF SIZE
}

\author{
Sakshi Jha ${ }^{1}$, Sachi Agrawal ${ }^{2}$, Chitesh Kumar Dewangan ${ }^{3}$ \\ ${ }^{1}$ Bachelors of Engineering, Department of Electronics and Telecommunication, Bhilai Institute of Technology, Durg, \\ India \\ ${ }^{2}$ Bachelors of Engineering, Department of Electronics and Telecommunication, Bhilai Institute of Technology, Durg, \\ India \\ ${ }^{3}$ Assistant Professor, Department of Electronics and Telecommunication, Bhilai Institute of Technology, Durg, India
}

\begin{abstract}
In this paper, an automated grading technique is presented. It sorts tomato fruit based on its size, using the digital image processing techniques. Quality examination of food and agricultural product are mostly sturdy and labour intensive in India. With the increased expectations for high nutrient and good standards, the necessity for correct, fast and objective quality determination of those characteristics in fruits continues to grow. Thus, we have sought to design a cost effective, high speed and efficient system to grade the tomatoes according to their size. The system will enable the non-destructive gradation of fruit, therefore enabling the user to get an efficient result which is not possible in case of manual inspection as human capabilities are affected by the tedium nature of the work, which results in reduced speed and efficiency. Moreover, the developed system will help in the areas where standardization is important.
\end{abstract}

Keywords: Grading, Tomato, Image processing, Food processing.

\section{INTRODUCTION}

Tomato is one of the most important protective food crops of India. It is grown in 0.458 Million hectare ( $\mathrm{M}$ ha) area with 7.277 Million metric ton (M mt) production and 15.9 metric ton/hectare (mt/ha) productivity [1]. In order to grade such an important and highly demanded fruit manual technique is not sufficient in terms of percentage accuracy and time.

Sorting of fruits and vegetables is one in every of the foremost necessary processes in fruits production, whereas this method is usually performed manually in most of the countries including India. The manual technique of handpicking the foremost effective fruit among the stock is also a time consuming methodology. Computer vision techniques have applied for evaluating food quality grading. So, for grading of fruits like tomato, orange, apple, mango, etc. this technique is able to be additional useful in trade to check the standard of fruits.

Thus we have designed a system to grade tomatoes based on their size. Size measurement is important for determining produce surface area. To determine the size we have used the threshold technique for grading the tomato as small, medium or large based on which end of the threshold they lies.

\subsection{Problem Identification}

Anuradha Gawande et al. [2] proposed a method to identify infected region from the input images and classify the infected patterns as per their level of infection aka low, average, medium, high, extreme high and fully infected fruits according to external surface. However, it processes only one image at a time rather than a bunch which is the major limitation of the system. Md. Rokunuzzaman et al. [3] considered only the defects on the tomato head as the basis for grading and for sorting neural network technique was applied. Some important parameters like color, size, and texture were not considered and for sorting a pre defined data base was required for comparison. P. Vimala Devi et al. [4] presented a review of various methods of fruit sorting and defect detection using machine vision technique. It also mentions the noise removal technique during the image processing method. Noise removal is one of the preprocessing steps that enhanced the image for further processing. Cheng-Jin Du et al. [6] published a review of the application of image processing techniques to different types of food products. It showed that various processing techniques have different degrees of success in different fields. CCD, MRI, Ultrasound, $\mathrm{CT}$ and ET sensor techniques are used for image acquisition of food. They also studied Statistical method, Fuzzy logic and Neural Network methods. Processing speed was a barrier in heavy duty real time applications, unable to handle large data streams.

\subsection{Motivation}

On reviewing various papers we were motivated to design a relatively simple system to grade tomatoes on the basis of their size as standardization is desired in the major markets of the world. Uniform size of the fruit in a package is a significant criteria for the consumers to buy the product. The paper presents the application of image processing technique to grade tomatoes on the size basis. 


\section{METHODOLOGY}

The paper aims at grading of tomatoes based on the size of the fruit. Pre-processing is done on a bunch of fruits mounted on top a system to remove the noise and crop out the individual fruits from the bunch.

Further the image is converted to a binary image and then the non-zero components of the image are calculated to give out the size of fruit in terms of area. The size ranges can be changed based on the size requirements.

\section{Algorithm}

The following algorithm is followed in the fruit grading method:

1. The process starts after the tomatoes are mounted on the platform.

2. A camera fixed atop the position of tomatoes captures its image.

3. The captured image is pre-processed to remove the image noise and cropped to separate images of all the tomatoes.

4. Then the color of the placed tomatoes is detected and graded good or bad based on the red color content on each tomato. The steps involved are:

A) The red component of the image is separated.

B) The acquired image is converted to a grayscale image.

C) The images of A and B are subtracted to get a new image.

D) The new image is filtered for noise components using median filter and converted to a binary image.

E) A function is called which returns the non-zero elements of the binary image. This value gives the area (size) of the fruit.

F) If area $>=21000$

Tomato is of Grade ' $A$ '

else if area $>=15000 \& \&$ area $<=20999$

Tomato is of Grade ' $\mathrm{B}$ '

else

Tomato is of Grade ' $\mathrm{C}$ '

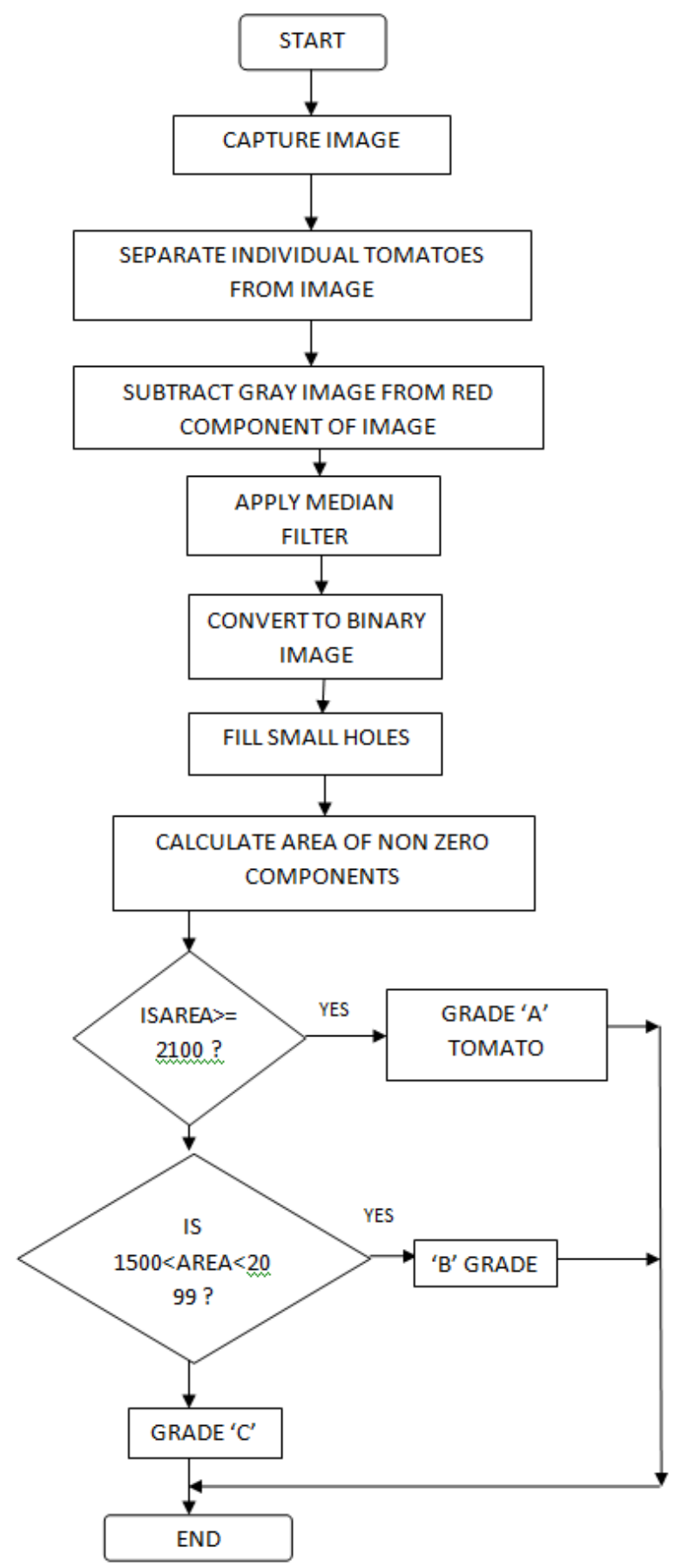

Fig 1: Flow chart of the grading process

\section{RESULT}

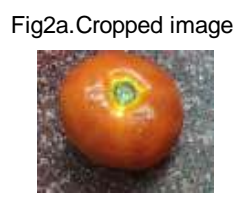

Fig2d.Binary image

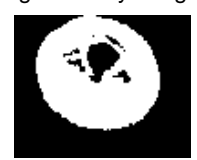

Fig2b.Subtracted image Fig2c.Median filtered image

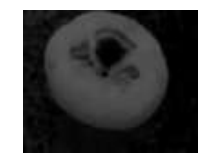

Fig2e. Holes filled

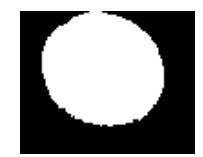

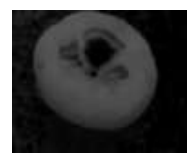

GradeA

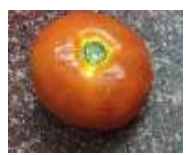

Fig 2. Grading of Grade A Tomato 


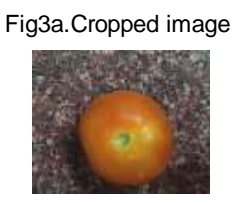

Fig3d.Binary image

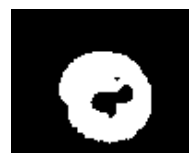

Fig3b.Subtracted image Fig3c.Median filtered image

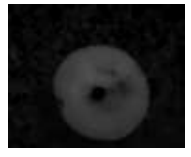

Fig3e. Holes filled

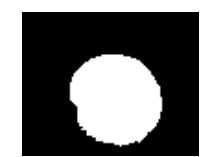

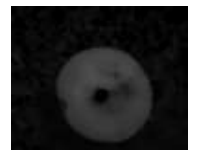

GradeC

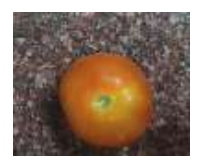

Fig 3. Grading of Grade C Tomato

The project grades the tomatoes based on its size. The figure above shows the steps involved in carrying out image detection and grading of tomato. Figure 1 shows a big size tomato and figure 2 shows a small size tomato. The experimental data is given below:

\begin{tabular}{|l|l|}
\hline Total tomatoes graded & 30 \\
\hline No. of good tomatoes present & 25 \\
\hline No. of good tomatoes detected & 05 \\
\hline
\end{tabular}

Thus the accuracy of the system is $84 \%$. The system is not affected by lighting as the non zero components are counted. The logic used is practical and experimentally found to be effective.

\section{CONCLUSION}

The proposed paper grades the tomatoes on the basis of size. The earlier paper proposed grading method of tomatoes on the basis of color which will be combined with the size along with the hardware to sort the tomatoes along with the grading. It will result in a smart system which will sort the tomatoes based on the grading done.

\section{REFERENCES}

[1] http://www.ncpahindia.com/tomato.php

[2] Anuradha Gawande, S. S. Dhande, "Implementation of Fruits Grading and Sorting System by Using Image

[3] Processing and Data Classifier", SSRG-IJCSE, volume 2, issue 6 June 2015.

[4] Md. Rokunuzzaman, H. P.W. Jayasuriya, "Development of a low cost machine vision system for sorting of tomatoes”, CIGR Journal, March, 2013 Agric Eng Int: CIGR Journal access Vol. 15, No.1.

[5] P. Vimala Devi, K. Vijayarekha, "Machine Vision Applications to Locate Fruits, Detect Defects and Remove Noise: A Review" RASAYAN J. Chem. Vol. 7, 2014.

[6] Chandra Sekhar, Bipan Tudu, Chiranjib Koley, "An Automated Machine Vision Based System for Fruit Sorting and Grading", IEEE 2012 Sixth International Conference on Sensing Technology (ICST).

[7] Cheng-Jin Du, Da-Wen Sun, "Recent Developments in the Applications of Image Processing Techniques for Food Quality Evaluation", Trends in Food Science and Technology 15, 2004.

[8] Hongshe Dang, Jinguo Song, Qin Guo, "A fruit size detecting and grading system based on image processing", 2nd International Conference on Intelligent Human Machine Systems And Cybernatics 2010.

[9] Chandra Sekhar, Bipan Tudu, Chiranjib Koley, "An Automated Machine Vision Based System for Fruit Sorting and Grading", IEEE 2012 Sixth International Conference on Sensing Technology (ICST).

[10] Sachi Agrawal, Sakshi Jha, Chitesh Dewangan, "Grading of tomatoes using digital image processing on the basis of color", IRJET, Volume 3, Issue 9, September 2016. 